\title{
Influence of Ferromagnetic Ribbon Width on $Q$ Factor and Magnetoelastic Resonance Frequency
}

\author{
S. Atalay*, O.O. Inan, V.S. Kolat and T. IzGi \\ Inonu University, Physics Department, Faculty of Science, Malatya, Turkey \\ Received: 07.12.2020 \& Accepted: 28.12.2020
}

Doi: 10.12693/APhysPolA.139.159

*e-mail: selcuk.atalay@inonu.edu.tr

\begin{abstract}
In this study, $\mathrm{Fe}_{40} \mathrm{Ni}_{38} \mathrm{Mo}_{4} \mathrm{~B}_{18}(2826 \mathrm{MB})$ amorphous ferromagnetic ribbons were used as a magnetoelastic sensor. The variation in resonance frequency and quality $(Q)$ factor values of samples with a $30 \mathrm{~mm}$ length and different widths is investigated as a function of a magnetic field. It is observed that as the sample width increases, the change in resonance frequency and the $Q$ factor both decrease. The parameter $Q \times$ amplitude/ $M$ is defined to understand the effect of a ribbon width on the resonance curve. According to this parameter, samples with 1 and $2 \mathrm{~mm}$ widths are found to have a better magnetoelastic effect.
\end{abstract}

topics: magnetoelastic sensor, resonance frequency, $Q$ factor

\section{Introduction}

The change in sample magnetisation under the influence of torque or stress applied to a ferromagnetic material is expressed as a magnetoelastic effect. Amorphous ferromagnetic magnetoelastic materials typically exhibit an extension or shrinkage of the order of $10^{-6}$ when exposed to an external magnetic field. This effect is known as magnetostriction [1]. Magnetostrictive materials convert magnetic energy into mechanical energy and vice versa. Accordingly, such materials are used in many application areas as magnetoelastic sensors (MESs). In general, magnetic materials with high magnetostriction values have been used as magnetoelastic sensors [2-5].

When magnetoelastic materials are exposed to a time-varying alternating current $(\mathrm{AC})$ magnetic field, they begin to vibrate along the applied field direction, that is, their length oscillates between shorter and longer. This vibration generates elastic waves and also leads to a change in the sample magnetisation. The signal produced by sample vibration can be detected by optical, acoustic, or magnetic techniques $[2,3]$. The sample vibration reaches a maximum when the field frequency of the applied AC magnetic field is equal to the ribbon's resonant frequency. The resonant frequency of such samples varies depending on the geometry, elastic coefficient, and mass (density) of the sample. Therefore, magnetoelastic sensors have been used as pressure [6], humidity [7, 8], temperature [9], liquid viscosity and density [10-12], chemical gasses [13], $\mathrm{pH}[14,15]$ and environmental parameters [16-22].
MESs are low-cost materials that can be easily shaped and produced in a variety of sizes. MESs also work wirelessly. These features make them attractive in a wide range of applications [2-5, 23].

The sensitivity of a MES is determined by a minimum frequency change that it can detect. This depends on the measurement system and the sharpness of the resonance curve, i.e., the quality factor ( $Q$ factor). So far, the effect of sample shapes and sizes on MESs has only been partially investigated. However, since there has been no detailed study of the effect of sample sizes on the magnetic field dependence of the $Q$ factor, in this article, the optimum sample sizes are determined by measuring the $Q$ factor values as a function of the magnetic field.

\section{Experimental}

We used a $\mathrm{Fe}_{40} \mathrm{Ni}_{38} \mathrm{Mo}_{4} \mathrm{~B}_{18}$ (2826MB) amorphous ferromagnetic ribbon as a MES and ribbons with a $30 \mathrm{~mm}$ length and $1,2,4,7$, and $10 \mathrm{~mm}$ widths were cut using a dice-sawing system for magnetoelastic measurements. Selected parameters of the $2826 \mathrm{MB}$ ribbon are given in Table I.

The magnetoelastic resonance measurement system is shown in Fig. 1. The amorphous ribbon is free to vibrate, with no clamp used to hold the sensor. The sensor was placed on the surface of the polished $\mathrm{Al}_{2} \mathrm{O}_{3}$ substrate and it was assumed that the friction is very small between the ribbon and the substrate. An AC magnetic field with a magnitude of $10 \mathrm{~A} / \mathrm{m}$ was applied along the ribbon length by the driver coil which leads to the vibration of the ribbon longitudinally and induces small voltages 


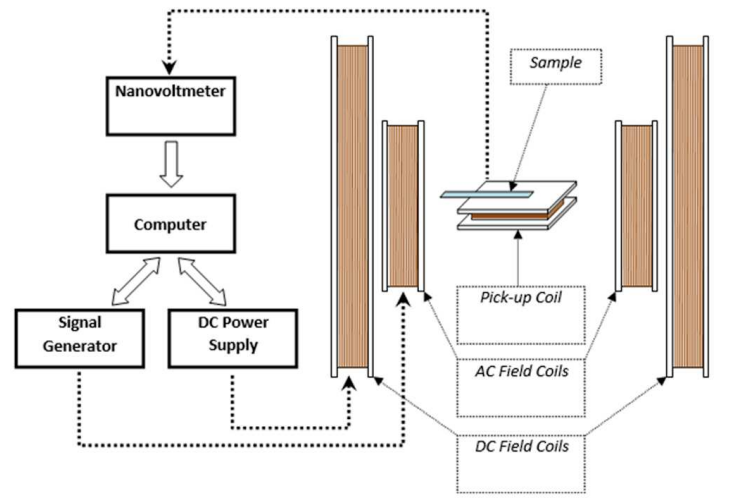

Fig. 1. Magnetoelastic measurement system.

TABLE I

Selected parameters of a $2826 \mathrm{MB}$ ribbon [24].

\begin{tabular}{l|c}
\hline \hline \multicolumn{1}{c|}{ Parameter } & Value \\
\hline saturation magnetostriction, $\lambda_{s}$ & $12 \mathrm{ppm}$ \\
magnetic permeability, $\mu$ & $>50000$ \\
saturation induction, $B_{\mathrm{s}}$ & $0.88 \mathrm{~T}$ \\
electrical resistivity, $R_{\mathrm{e}}$ & $138 \mu \Omega \mathrm{cm}$ \\
density, $\rho$ & $7.90 \mathrm{~g} / \mathrm{cm}^{3}$ \\
elastic modulus, $E$ & $100-110 \mathrm{GPa}$ \\
Curie temperature, $T_{\mathrm{C}}$ & $353^{\circ} \mathrm{C}$ \\
coupling factor, $k$ & 0.98
\end{tabular}

in the pick-up coil. The pick-up coil was placed perpendicular to the $\mathrm{AC}$ magnetic field direction (see Fig. 1). In addition, the base signal formed in the coil without the sample was recorded by the software and during the measurement performed with the sample, this base signal was extracted by the software, i.e., the measured signal is the signal originating from the sample. The pick-up coil has the dimensions of $6 \times 6 \mathrm{~cm}^{2}$ and 330 turns of a copper wire. A Stanford $345 \mathrm{AC}$ function generator was used to produce an AC magnetic field and the signal was amplified using an Accel TS200 amplifier. The system was fully computer controlled. The frequency of the signal generator was swept by the software. At each frequency value, the induced signal amplitude was read from a Keithley 2000 multimeter which was connected to the pick-up coil. The software determined the resonance frequency by finding the frequency where the amplitude is at a maximum. An external direct current magnetic field was applied using a bipolar Kepco power supply using Helmholtz coils. Earth's magnetic field was cancelled out using the Helmholtz coil system.

\section{Results and discussion}

Figure 2 shows the resonance curves of the MES with a $7 \mathrm{~mm}$ width obtained at different magnetic field values. Resonance frequency measurements were made at four different magnetic field

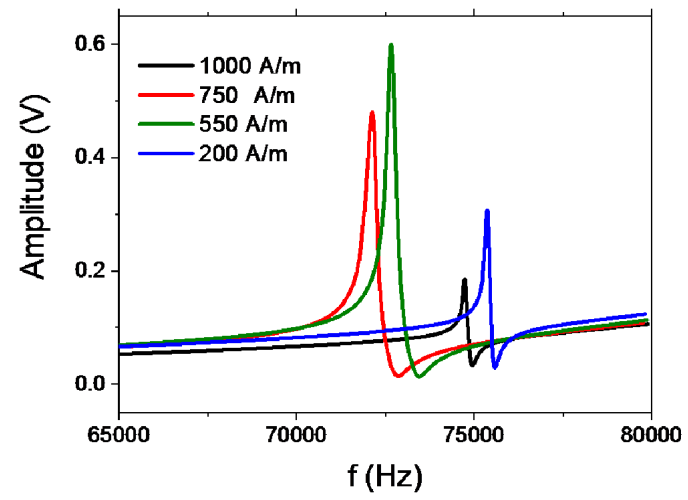

Fig. 2. Variation of resonance peak values at various magnetic field values for a ribbon with a $7 \mathrm{~mm}$ width.

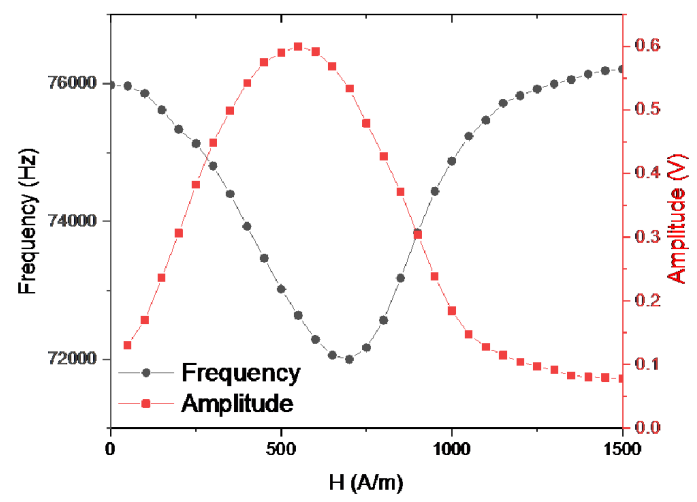

Fig. 3. Change of the resonance frequency and amplitude of a ribbon with a $7 \mathrm{~mm}$ width as a function of the applied magnetic field.

values to show the resonance curve behaviour in the whole range of the magnetic field change. The first and second curves were measured at low magnetic field values of $H=200 \mathrm{~A} / \mathrm{m}$ and $550 \mathrm{~A} / \mathrm{m}$, respectively, and the maximum change in the amplitude was observed at $550 \mathrm{~A} / \mathrm{m}$. The third and fourth curves were obtained at high magnetic field values of $H=750 \mathrm{~A} / \mathrm{m}$ and $1000 \mathrm{~A} / \mathrm{m}$, respectively. It can be seen that the magnetic field has a significant influence on the resonance frequency value and resonance curve shape.

The resonance frequency and vibration amplitude change as a function of the magnetic field are given in Fig. 3 for the ribbon with a $7 \mathrm{~mm}$ width. Young's modulus $E$ of the ribbon is related to the resonance frequency of ribbon by $E=4(L f)^{2} \rho[25]$, where $\rho$ is the sample density and $L$ is the sample length of the ribbon.

Figure 4 shows the normalised Young modulus of ribbons with widths of $1,2,4,7$ and $10 \mathrm{~mm}$. The $E / E_{s}$ curves were obtained based on the relation $E(H) / E_{s}=\left(f(H) / f_{s}\right)^{2}$, where $f(H)$ is the resonance frequency at an $H$ value of magnetic field, $f_{s}$ is the resonance frequency at saturation ( $\approx 1500 \mathrm{~A} / \mathrm{m}$ in our measurements), $E(H)$ is 


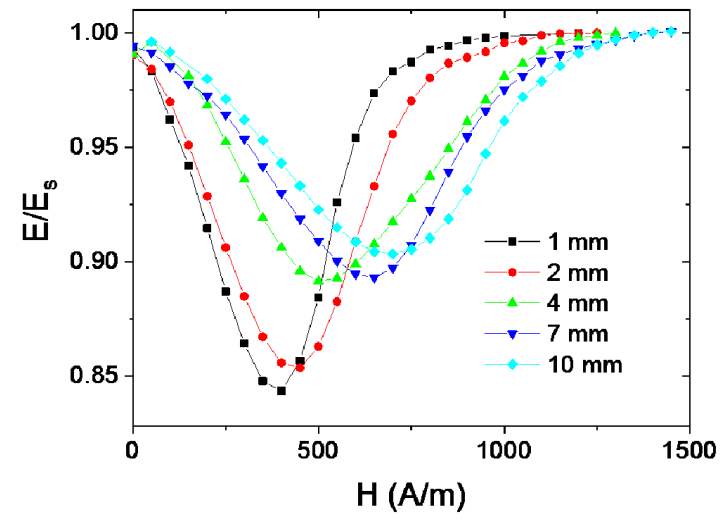

Fig. 4. Normalised Young's modulus of ribbons with different sample widths.

Young's modulus at $H$ and $E_{s}$ is Young's modulus at saturation. Figure 4 shows that the magnitude of $E_{\min } / E_{s}$ decreases with increasing ribbon width and it changes from 0.843, 0.856, 0.890, 0.893 and 0.903 for samples widths of $1,2,4,7$ and $10 \mathrm{~mm}$, respectively.

The magnetic field dependence of $E$, or in other words, the $\Delta E$ effect, was discussed by Livingston [26] who proposed a simple model of the rotation of magnetic moments. The model considers an amorphous ribbon that is annealed in a magnetic field parallel to the ribbon width to produce a magnetic easy axis in this direction. The model assumes that the magnetisation process is only due to the magnetic moment rotation, where a longitudinal magnetic field would rotate the magnetic moments from the width direction towards the longitudinal direction. The magnetic field dependence of Young's modulus is given by the expression

$$
\frac{E(H)}{E_{s}}=\left(1+\frac{9 \lambda_{s}^{2} E_{s} H^{2}}{\mu_{0} M_{s} H_{k}^{3}}\right)^{-1},
$$

where $H$ is the applied magnetic field, $M_{s}$ is the saturation magnetisation, $\lambda_{s}$ is the saturation magnetostriction and $H_{k}$ is the anisotropy field given by $2 K /\left(\mu M_{s}\right)$ with the total anisotropy constant $K$. Considering the Livingston model and the $\mathrm{V}$ shape of the $E / E_{s}$ versus the magnetic field curve, it can be concluded that the amorphous ribbon has a magnetic easy axis that is perpendicular to the ribbon length. Moreover, according to the Livingston model, the magnitude of $E / E_{s}$ is determined by $M_{s}, \lambda_{s}$ and $K$. Since we use the same ribbon and no annealing treatments were performed, $M_{s}$ and $\lambda_{s}$ are not changed in our case. The parameter that is changed is the total anisotropy energy. Now, keeping all the other parameters for our ribbon the same, it turns out that only the shape anisotropy change affects the total $K$. The variation of $K$ was also confirmed by the variation in $H_{k}$ which is determined in the minimum of the $E / E_{s}$ curve. The measurements of $H_{k}$ showed 385, 450, 510, 650 and $700 \mathrm{~A} / \mathrm{m}$ for ribbons with widths of $1,2,4,7$ and $10 \mathrm{~mm}$, respectively. As has been reported [25], the main

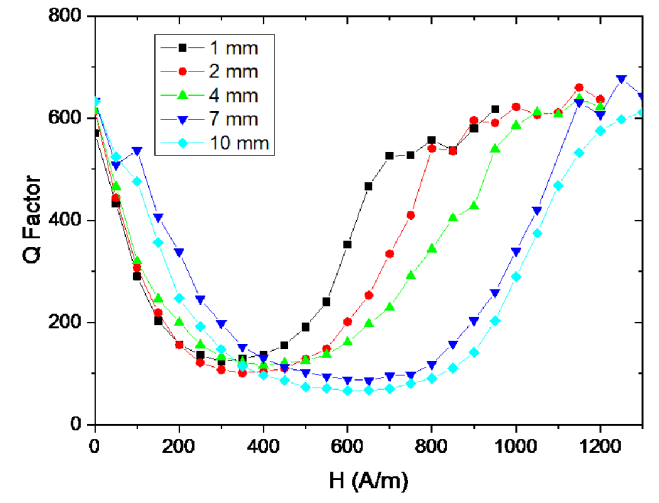

Fig. 5. Magnetic field dependence of $Q$ factor.

reason for the change in shape anisotropy is the demagnetisation factor which increases with increasing ribbon width, leading to a decrease in the magnitude of $E / E_{s}$.

The sharpness of the resonance curves can be determined by the $Q$ factor. In Fig. 5, the typical resonance curves with low and high $Q$ factor values are shown. A sharper curve means a high $Q$ factor and a broad resonance curve means a low $Q$ factor. Therefore, to basically measure the minimum frequency change, we need a sample with a high $Q$ factor. Generally, the $Q$ factor is determined from

$$
Q=\frac{f_{r}}{\Delta f},
$$

where $f_{r}$ is the peak value of the resonance curve, as shown in Fig. 5, and $\Delta f$ is the frequency range between the two frequencies where the observed signal's power is half the size of the maximum power at the resonance frequency. Kaczkowski and Malkinski [27] proposed a slightly different approach to the calculation of the $Q$ factor

$$
Q=\frac{f_{r}}{f_{m}^{\prime}-f_{M}^{\prime}},
$$

where $f_{m}^{\prime}$ is the minimum and $f_{M}^{\prime}$ is the maximum at resonance frequency. A detailed study regarding the calculation method for the $Q$ factor was given by Lopes et al. [28]. They calculated the $Q$ factor using (2) and (3) and also from numerical fitting to the resonance curve. They found that the $Q$ factor determined from (2) is slightly higher than the $Q$ factor obtained from (3) and numerical fitting. They also found that the $Q$ factor values obtained from (3) and numerical fitting are nearly the same. Numerical fitting is a long process and requires significant work. In contrast, the calculation of the $Q$ factor using (3) is much easier and we therefore adopted this approach. The magnetic field dependence of the $Q$ factor with different ribbon widths is given in Fig. 5. The magnetic field dependence of the $Q$ factor of all samples shows similar curves. It is observed that the $Q$ factor values first started to decrease and then increased again after reaching a minimum value. After reaching high magnetic field values, it was found that the $Q$ factor 


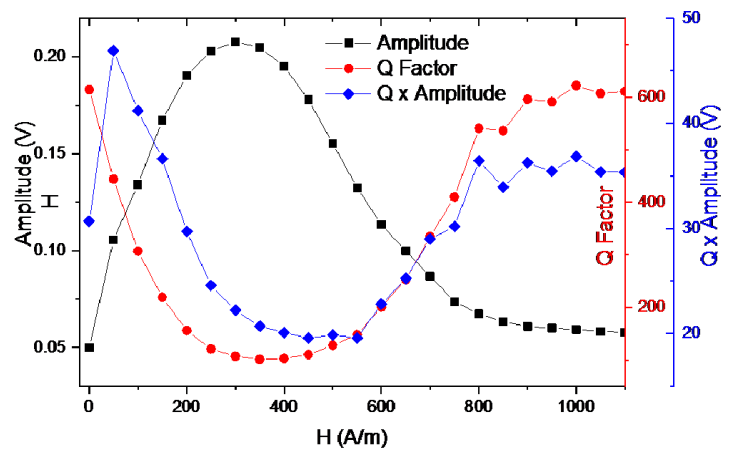

Fig. 6. Effect of magnetic field on amplitude, $Q$ factor and $Q \times$ amplitude for a sample with a $2 \mathrm{~mm}$ width.

values did not significantly change. The $Q$ factor values were found to reach the minimum value more quickly in samples with a width of 1 and $2 \mathrm{~mm}$ and then began to increase at lower magnetic field values than samples with other larger widths. As the width of the ribbon increases, it is observed that the $Q$ factor reaches lower values and the sample with the lowest $Q$ factor value is the ribbon with a width of $10 \mathrm{~mm}$. In addition, it was found that the $Q$ values did not change much in the magnetic field region of 450 to $750 \mathrm{~A} / \mathrm{m}$ in samples with large widths, such as 7 and $10 \mathrm{~mm}$. The results are in agreement with the previously reported studies. Sagasti et al. [25] also studied the effect of a sample size and they found that the magnitude of $E / E_{s}$ increases as the sample width decreases and the $Q$ factor decreases as the sample width increases which is fully in agreement with Figs. 4 and 5.

A high $Q$ factor and amplitude are required for a MES design. However, the results show that the $Q$ values are low when the signal amplitude is large (see Figs. 2, 3 and 5). For this reason, we defined a statement - the $Q \times$ amplitude - to discover which magnetic field of both the $Q$ factor and the resonance signal amplitude can obtain more suitable values (see Fig. 6). It is seen that in the magnetic ribbons with 1 and $2 \mathrm{~mm}$ widths, this magnetic field value is $\approx 50 \mathrm{~A} / \mathrm{m}$ and this value shifts to larger values, such as $200-250 \mathrm{~A} / \mathrm{m}$ in samples with 7 and $10 \mathrm{~mm}$ widths.

It is well known that one of the important factors in magnetoelastic design is the sensor's initial mass $M$. According to $\Delta f=-(\Delta m / M) \times f$, where $f$ is the resonance frequency, $\Delta f$ is the change in the resonance frequency and $\Delta m$ is the change in the mass of the sensor, the amount of mass collected on the sample that is to be detected is proportional to $1 / M$ and a larger $M$ means a less sensitive magnetoelastic sensor. Therefore, we go one step further to consider the effect of the initial masses of magnetoelastic sensors with different widths. As shown in Fig. 7, we can redraw the results according to the statement $Q \times$ amplitude $/ M$. As can be seen, the sample with a $1 \mathrm{~mm}$ width has

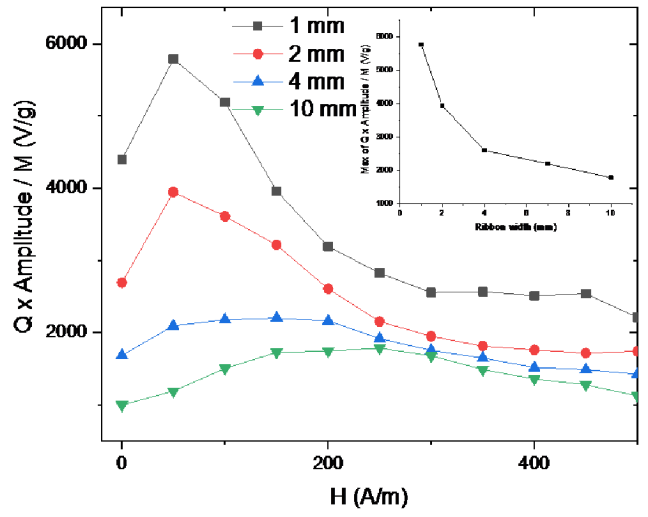

Fig. 7. Magnetic field dependence of $Q \times$ amplitude $/ M$ for a ribbon with various sample widths.

a large value of $Q \times$ amplitude/ $M$ that increases with increasing magnetic field and reaches a maximum of $5789 \mathrm{~V} / \mathrm{g}$. The inset in Fig. 7 shows that the max value of $Q \times$ amplitude/ $M$ decreases nearly exponentially with increasing ribbon width. The results show that a sample width smaller than $2 \mathrm{~mm}$ gives rise to a better $Q \times$ amplitude $/ M$ value and a ribbon width of less than $2 \mathrm{~mm}$ was considered sufficient to obtain accurate magnetoelastic resonance measurements.

\section{Conclusions}

The variation of resonance frequency and $Q$ factor values of $\mathrm{Fe}_{40} \mathrm{Ni}_{38} \mathrm{Mo}_{4} \mathrm{~B}_{18}$ amorphous ferromagnetic ribbons with a $30 \mathrm{~mm}$ length and various widths have been analysed extensively as a function of the magnetic field. It was found that the magnitude of $E_{\min } / E_{s}$ decreases with increasing ribbon width and it changes from $0.843,0.856,0.890$, 0.893 and 0.903 for $1,2,4,7$ and $10 \mathrm{~mm}$ sample widths, respectively. This variation in $E_{\min } / E_{s}$ is due to the change in the shape anisotropy and consequently the change in the demagnetisation factor which increases with increasing ribbon width, leading to a decrease in the magnitude of $E / E_{s}$.

It was observed that the $Q$ factor values first started to decrease and then increased again after reaching a minimum value and they increased again as a function of the magnetic field. The $Q$ factor values were found to reach the minimum value more quickly in samples with a width of 1 or $2 \mathrm{~mm}$ and then began to increase at lower magnetic field values than samples with other larger widths. To understand the effect of the sample mass and its width in more detail, a parameter given with the expression $Q \times$ amplitude/ $M$ was defined. Since in our measurements, the ribbon density and thickness are the same, $M$ is also the indication of the surface area. According to this parameter, samples with 1 and $2 \mathrm{~mm}$ widths were found to have a better magnetoelastic effect. 
It has been shown that the sample width has a significant influence on the magnetic field dependence of the resonance frequency or Young's modulus and $Q$ factor. We deduce that samples with a width smaller than $2 \mathrm{~mm}$ for a $30 \mathrm{~mm}$ length are required for a good magnetic and magnetoelastic material response which is also shown in our measurements and previous studies.

\section{Acknowledgments}

This work was supported by TUBITAK with project number MFAG-119F144.

\section{References}

[1] M.R.J. Gibbs, Phys. Scr. T45, 115 (1992).

[2] C.A. Grimes, C.S. Mungle, Z.F. Zeng, M.K. Jain, W.R. Dreschel, M. Paulose, K.G. Ong, Sensors 2, 294 (2002).

[3] J.J. Beato-Lopez, G.G. UrdanizVillanueva, J.I. Perez-Landazabal, C. Gomez-Polo, Materials 13, 2175 (2020).

[4] X. Guo, J.Z. Wang, Y.J. Zhao, R. Liu, Q. Zhang, Z.Y. Yuan, S.B. Sang, Biochem. Eng. J. 156, 107498 (2010).

[5] S. Atalay, V.S. Kolat, F.E. Atalay, N. Bayri, H. Kaya, T. Izgi, J. Magn. Magn. Mater. 465, 151 (2018).

[6] D. Kouzoudis, C.A. Grimes, Smart Mater. Struct. 9, 885 (2000).

[7] C.A. Grimes, D. Kouzoudis, Sens. Actuat. A Phys. 84, 205 (2000).

[8] S. Atalay, T. Izgi, V.S. Kolat, S. Erdemoglu, O.O. Inan, Sensors 20, 425 (2020).

[9] M.K. Jain, S. Schmidt, K.G. Ong, C. Mungle, C.A. Grimes, Smart Mater. Struct. 9, 502 (2000).

[10] M.K. Jain, S. Schmidt, C.A. Grimes, Appl. Acoust. 62, 1001 (2001).

[11] D. Kouzoudis, C.A. Grimes, J. Appl. Phys. 87, 6301 (2000).

[12] S. Atalay, V.S. Kolat, N. Bayri, T. Izgi, J. Supercond. Nov. Magn. 29, 1551 (2016).
[13] Q.Y. Cai, M.K. Jain, C.A. Grimes, Sens. Actuat. B Chem. 77, 614 (2001).

[14] C. Ruan, K.G. Ong, C. Mungle, M. Paulose, N.J. Nickl, C.A. Grimes, Sens. Actuat. B Chem. 96, 61 (2003).

[15] P. Pang, X. Gao, X. Xiao, W. Yang, Q. Cai, S. Yao, Anal. Sci. 23, 463 (2007).

[16] J. Wan, M.L. Johnson, R. Guntupalli, V.A. Petrenko, B.A. Chin, Sens. Actuat. $B$ Chem. 127, 559 (2007).

[17] S.J. Huang, Y.J. Wang, Q.Y. Cai, J.D. Fang, Chin. J. Anal. Chem. 38, 105 (2010).

[18] L.V.R. Beltrami, S.R. Kunst, E.J. Birriel, C.D. Malfatti, Thin Solid Films 624, 83 (2017).

[19] X. Guo, S. Gao, S.B. Sang, A.Q. Jian, Q.Q. Duan, J.L. Ji, W.D. Zhang, Biosens. Bioelectron. 82, 127 (2016).

[20] J.C. Yin, Y.S. Wang, B. Zhou, X.L. Xiao, J.H. Xue, J.C. Wang, Y.S. Wang, Q.M. Qian, Sens. Actuat. B Chem. 188, 147 (2013).

[21] W. Shen, L.C. Mathison, V.A. Petrenko, B.A. Chin, J. Phys. D Appl. Phys 43, 015004 (2010).

[22] A.L. Possan, C. Menti, M. Beltrami, A.D. Santos, M. Roesch-Ely, F.P. Missell, Mater. Sci. Eng. C 58, 541 (2016).

[23] S. Atalay, V.S. Kolat, T. Izgi, Acta Phys. Pol. A 136, 531 (2019).

[24] L. Pen, K. Yu, Y. Tan, Materials 12, 1135 (2019).

[25] A. Sagasti, J. Gutierrez, A. Lasheras, J.M. Barandiaran, Sensors 19, 4296 (2019).

[26] J.D. Livingston, Phys. Status Solidi A 70, 591 (1982).

[27] Z. Kaczkowski, L. Malkinski, J. Magn. Magn. Mater. 41, 343 (1984).

[28] A.C. Lopes, A. Sagasti, A. Lasheras, V. Muto, J. Gutierrez, D. Kouzoudis, J.M. Barandiaran, Sensors 18, 887 (2018). 